\title{
Infective Endocarditis with Unusual Location of Vegetation in a Patient with Hemodialysis Followed by Recurrent Catheter-Related Blood Stream Infection
}

\author{
Ho Min Yong, Duk Won Bang, Byoung Won Park, Min Ho Lee, Tae Hyong Kim, Jae Hee Han \\ Department of Internal Medicine, Soonchunhyang University Seoul Hospital, Soonchunhyang University College of Medicine, Seoul, Korea
}

\begin{abstract}
The incidence of infective endocarditis (IE) in hemodialysis patients is higher than the general population. Hemodialysis patients with IE have a poorer prognosis than other patients with IE. The most common sites of IE in hemodialysis patients are the mitral valve and aortic valve. A 47-year-old woman had suffered from recurrent catheter-related blood stream infection and she was strongly suspected of IE. Despite repeated transthoracic echocardiography and transesophageal echocardiography (TEE), IE was revealed several months later by TEE due to the unusual location of the vegetation. The vegetation was detected on the left ventricle outflow tract. She recovered well without any complication after mitral valve replacement surgery.
\end{abstract}

Keywords: Endocarditis; Renal dialysis; Catheter-related infections

\section{INTRODUCTION}

Infective endocarditis (IE) is one of the most serious infectious complications among patients undergoing hemodialysis. The incidence of IE in hemodialysis patients is much higher than the general population [1]. In addition, hemodialysis patients who develop IE have a poorer prognosis and higher mortality than other patients with IE [1-3]. The most common sites of IE in hemodialysis patients are the mitral valve (MV) and aortic valve [2]. We report an interesting case of infective endocarditis that was difficult to detect due to the unusual location of the vegetation at the left ventricular outflow tract.

\section{CASE REPORT}

A 47-year-old female was admitted with fever developed several days previously. She had a medical history of diabetes mellitus, hypertension, and end stage renal disease with a 10 -year history of hemodialysis. On physical examination, fever with a temperature of $38^{\circ} \mathrm{C}$ was evident, blood pressure was $120 / 80 \mathrm{~mm} \mathrm{Hg}$, and pulse rate was 72 per minute. A central venous tunneled catheter was in- serted in the left femoral vein for dialysis. The exit site of central venous catheter showed redness with purulent discharge. In the past, she had been hospitalized in Soonchunhyang University Seoul Hospital three times (3, 5, and 9 months previously) because of catheter-related blood stream infection (CRBSI). At the first admission for CRBSI, she had visited the emergency department for relief of a fever that had developed one day before. At that time she had been undergoing hemodialysis via right internal jugular vein tunneled catheter because right upper arm arteriovenous bridge graft (AVBG) was ruptured one month ago. The right internal jugular vein catheter was removed and temporary right femoral catheter was inserted. The result of blood culture and culture for discharge on catheter exit site was positive for methicillin-sensitive Staphylococcus aureus (MSSA). Transthoracic echocardiography (TTE) showed normal systolic function, no regional wall motion abnormality, trace mitral regurgitation, and trace tricuspid regurgitation with mild pulmonary hypertension. No vegetation was detected by TTE. Vancomycin (1,250 mg per 48 hours) was administered via intravenous route initial 2 days and cefazolin $(1 \mathrm{~g}$ every 12 hours, intravenously) was administered for 2 weeks after MSSA was identified from blood and discharge culture. The oper- 

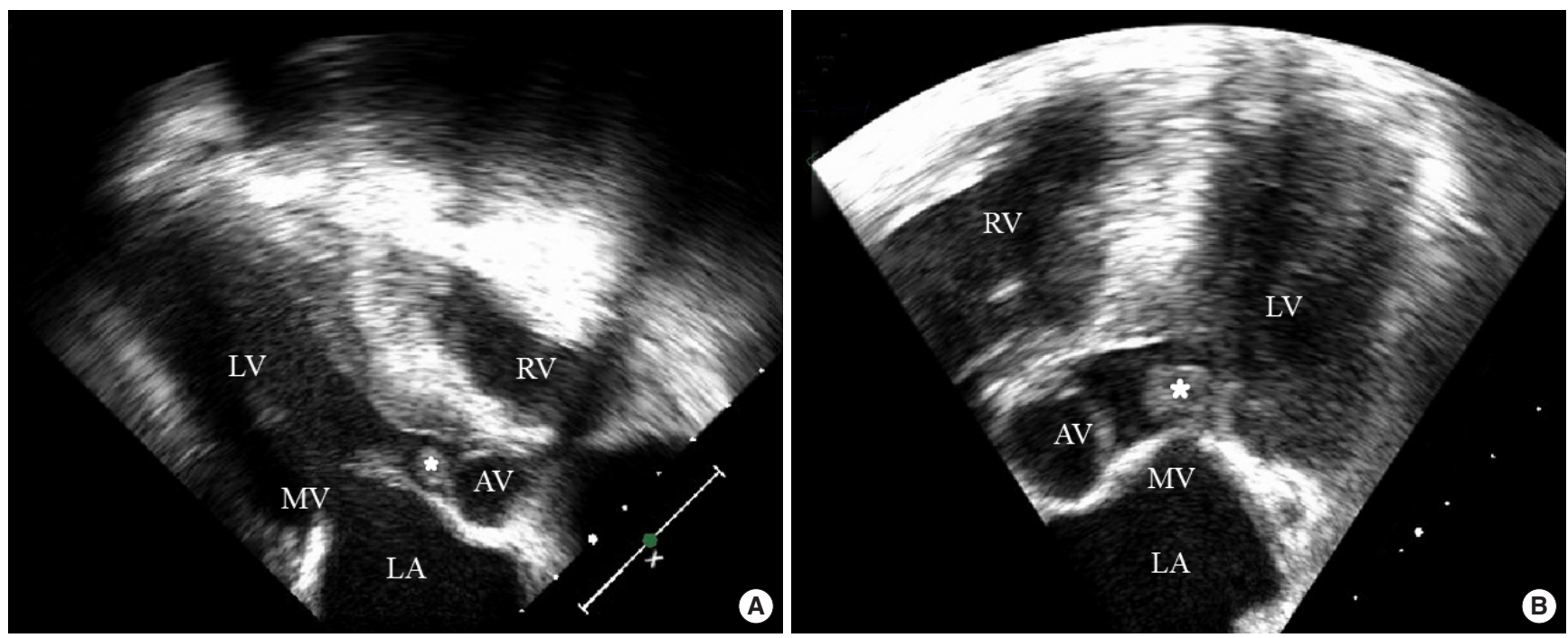

Fig. 1. Transesophageal echocardiography demonstrated a vegetation $\left(^{*}\right)$ on left ventricle outflow tract between non-coronary cusp of aortic valve and ventricular side of anterior mitral valve leaflet. (A) Mid esophageal three chamber view (120 degree). (B) Mid esophageal five chamber view (0 degree). LA, left atrium; LV, left ventricle; $\mathrm{RV}$, right ventricle; $\mathrm{MV}$, mitral valve; $\mathrm{AV}$, aortic valve.

ation for the new vascular access was planned after she recovered from bacteremia, but the patient refused the operation due to the economical problem. Four months later, she developed fever again. The patient had maintained a left internal jugular vein tunneled catheter for dialysis, and the exit site of catheter was shown purulent discharge. The result of blood culture and culture of catheter tip was positive for MSSA again. A transesophageal echocardiography (TEE) was performed, but no vegetation was seen. Cefazolin was administered again with same dose for 2 weeks. She became asymptomatic and was discharged. Two months later, she visited the hospital for fever. She had been maintained hemodialysis via right femoral vein tunneled catheter and the exit site of catheter had shown redness and purulent discharge. The catheter was removed and new hemodialysis tunneled catheter was inserted in left femoral vein. The result of blood and discharge culture was positive for MSSA again. Intravenous vancomycin was administered 3 days and then cefazolin ( 1 g every 12 hours, intravenously) was administered after MSSA was identified from the culture studies. Three months later from the third episode of CRBSI, she developed fever again. Because CRBSI was suspected, the catheter was removed immediately. The operation for the new vascular access had been planned many times after rupture of AVBG and the operation could not be performed because of recurrent infection and poor compliance of patient. Vancomycin (1,250 mg per 48 hours) was administered intravenously because $S$. aureus could be responsible for the persistent fever. Blood culture was positive for
MSSA 3 days later. Appropriately, vancomycin was deescalated to intravenous nafcillin ( 2 g per 24 hours). However, fever was sustained until day 5 of antibiotic administration. Since the possibility of infective endocarditis was strongly suggested, a repeated TEE was advised by an infectious diseases consultant. The TEE revealed a $0.7 \times 0.7-\mathrm{cm}$-sized vegetation on the left ventricle outflow tract (LVOT) between the non-coronary cusp of the aortic valve and the ventricular side of the anterior MV leaflet (Fig. 1). Despite adequate dose and route of administration of antibiotic for an additionally 10 days, the fever was not controlled and the size of the vegetation was enlarged to more than $1 \mathrm{~cm}$ (Fig. 2). An elective mitral valve replacement (MVR) surgery was performed. A $1 \times 2-\mathrm{cm}-$ sized vegetation was attached with MV lateral commissure to the P1 chordae with no involvement of the aortic valve. Two weeks after MVR, her vital signs stabilized without fever. She underwent repeat TEE, which showed bioprosthetic valve without dysfunction (Fig. 3). Nafcillin (2 g per 4 hours) was administered intravenously for 4 weeks after surgery. She has been doing well during 6 months follow-up and follow-up TEE after 6 months from surgery of MVR demonstrated no valvular dysfunction without vegetation (Fig. 4).

\section{DISCUSSION}

Dialysis is a widely recognized risk factor for IE. The incidence of IE in dialysis patients is estimated at 308/10,000 patient-years, 

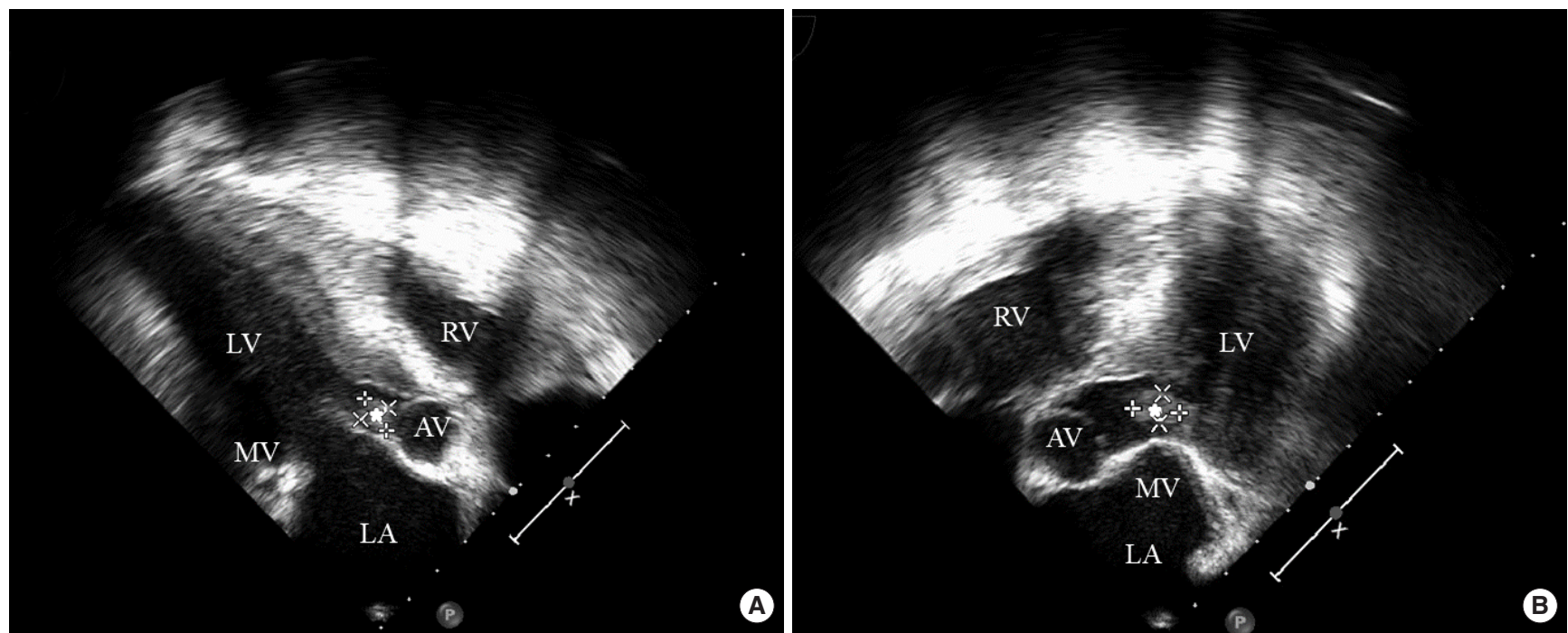

Fig. 2. The size of vegetation (*) was enlarged to $1.2 \times 0.7 \mathrm{~cm}$ on the transesophageal echocardiography 1 week later from initial transesophageal echocardiography. (A) Mid esophageal three chamber view (120 degree). (B) Mid esophageal five chamber view (0 degree). LA, left atrium; LV, left ventricle; RV, right ventricle; MV, mitral valve; $\mathrm{AV}$, aortic valve.

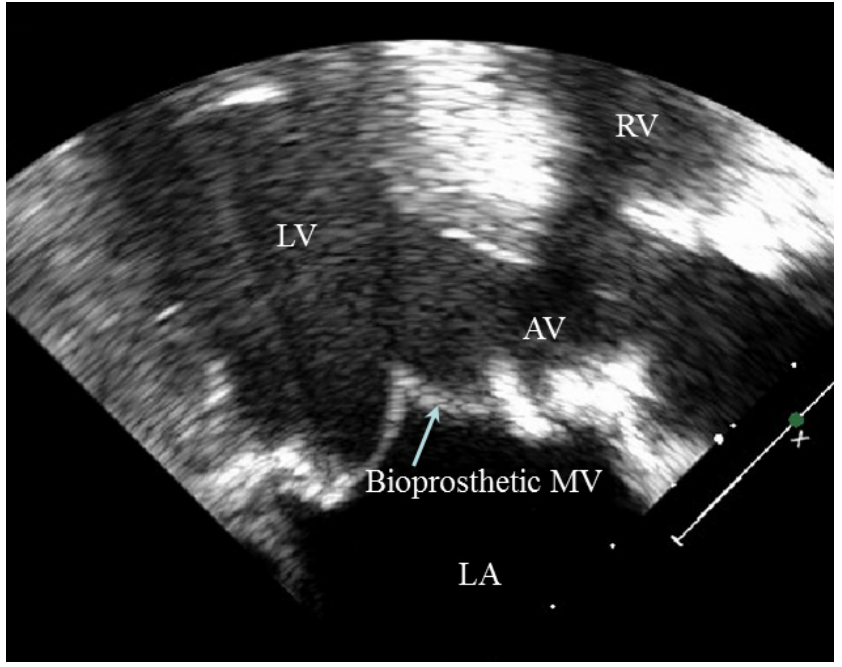

Fig. 3. Follow-up transesophageal echocardiography in mid esophageal three chamber view (120 degree) after mitral valve replacement demonstrated bioprosthetic valve without dysfunction (arrow). LA, left atrium; LV, left ventricle; $\mathrm{RV}$, right ventricle; $\mathrm{MV}$, mitral valve; $\mathrm{AV}$, aortic valve.

which is 50 to 180 times higher compared to the general population $[1,4]$. In addition, the prognosis of IE in hemodialysis patients is poor. The mortality rate among dialysis patients with IE ranges from $30 \%$ to $56 \%$ at 1 year [4] and in-hospital mortality is twice the mortality for IE in the general population $[2,3,5]$. This increased risk for IE in hemodialysis patients is attributable to transient bacteremia resulting from repetitive vascular access through an arteriovenous fistula or indwelling catheter [6,7]. A combina-

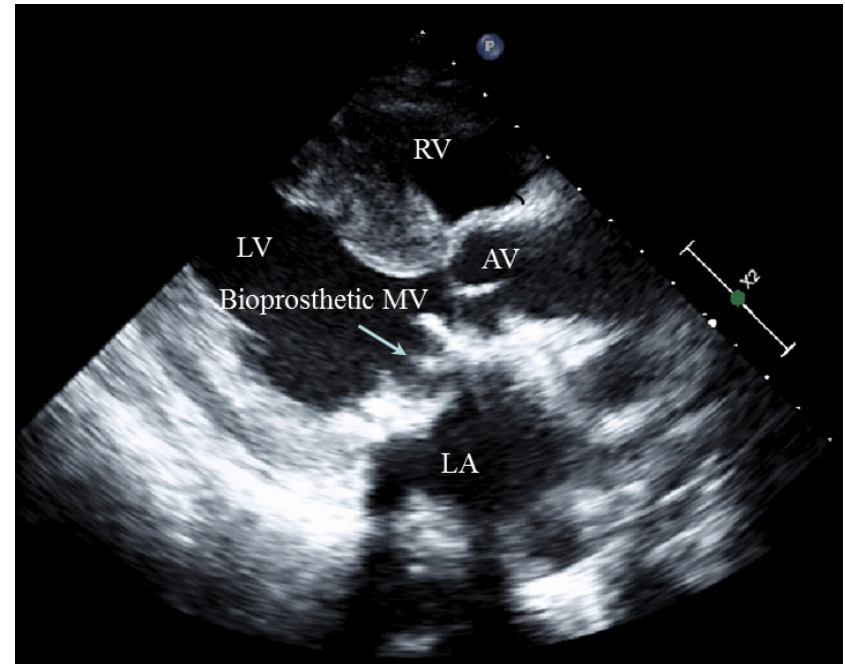

Fig. 4. Follow-up transthoracic echocardiography in mid esophageal three chamber view (120 degree) on final admission after 6 months from surgery of mitral valve replacement and vegetation removal (arrow). LA, left atrium; LV, left ventricle; RV, right ventricle; $\mathrm{MV}$, mitral valve; $\mathrm{AV}$, aortic valve.

tion of valvular heart disease [8] and impaired immunity secondary to uraemia [9] contribute to the increased risk for IE. The incidence of bacteremia in hemodialysis patients is dependent on the type of vascular access. The patients with indwelling catheter have higher incidence of bacteremia and poorer prognosis compared to the patients with arteriovenous fistula $[9,10]$.

In the majority of cases, IE in hemodialysis patients affects the mitral and aortic valves [1-3]. Like our case, IE on the left ventricu- 
lar outflow tract is rare and only few cases have been reported in the literature $[11,12]$. Two cases of IE with vegetation on the LVOT already had a history of aortic regurgitation, which may have contributed to damaging the LVOT endocardium over the years. In our case, the patient had no valvular heart disease except MV annulus calcification. She had suffered from recurrent CRBSI, but IE was revealed by TEE several months later. She might have had IE earlier, but vegetation was not detected by previous TTE or TEE, due to the unusual location of the vegetation. Thus, if a patient is strongly suspected of IE and vegetation is not detected by TTE or TEE, physicians need to be more cautious and meticulous because occasionally vegetation can be detected unusual location as our case. The indications for surgery in patients with IE include refractory heart failure, recurrent emboli, paravalvular extension of infection with development of abscess and/or fistula, and persistent infection despite appropriate antibiotics $[2,13]$. Early valve replacement improves survival rate compared to no surgery [1,2]. In our case, the patient had persistent fever despite sufficient antibiotic therapy. Thus, MVR was performed.

We report this interesting case because the unusual location of the vegetation made the diagnosis of IE difficult, despite repeated TTE and TEE in a hemodialysis patient who suffered recurrent MSSA bacteremia.

\section{REFERENCES}

1. Jones DA, McGill LA, Rathod KS, Matthews K, Gallagher S, Uppal R, et al. Characteristics and outcomes of dialysis patients with infective endocarditis. Nephron Clin Pract 2013;123:151-6.

2. Kamalakannan D, Pai RM, Johnson LB, Gardin JM, Saravolatz LD. Epidemiology and clinical outcomes of infective endocarditis in hemodialysis patients. Ann Thorac Surg 2007;83:2081-6.

3. Seo SW, Kim TH, Hyon MS, Choo EJ, Jeon MH, Moon C, et al. Characteristics of infective endocarditis in 4 university hospitals where Staphylococcus aureus is the most common causative organism. Infect Chemother 2008;40:316-22.

4. Abbott KC, Agodoa LY. Hospitalizations for bacterial endocarditis after initiation of chronic dialysis in the United States. Nephron 2002;91:203-9.

5. Spies C, Madison JR, Schatz IJ. Infective endocarditis in patients with end-stage renal disease: clinical presentation and outcome. Arch Intern Med 2004;164:71-5.

6. Marr KA, Sexton DJ, Conlon PJ, Corey GR, Schwab SJ, Kirkland KB. Catheter-related bacteremia and outcome of attempted catheter salvage in patients undergoing hemodialysis. Ann Intern Med 1997;127:275-80.

7. Sexton DJ. Vascular access infections in patients undergoing dialysis with special emphasis on the role and treatment of Staphylococcus aureus. Infect Dis Clin North Am 2001;15:731-42, vii.

8. Umana E, Ahmed W, Alpert MA. Valvular and perivalvular abnormalities in end-stage renal disease. Am J Med Sci 2003;325:237-42.

9. Minnaganti VR, Cunha BA. Infections associated with uremia and dialysis. Infect Dis Clin North Am 2001;15:385-406, viii.

10. Rehman R, Schmidt RJ, Moss AH. Ethical and legal obligation to avoid long-term tunneled catheter access. Clin J Am Soc Nephrol 2009;4:456-60.

11. Quah BS, Selladurai BM, Jayakumar CR, Mahendra Raj S. Left ventricular outflow tract (LVOT) vegetations and spontaneous obliteration of a large ruptured intracranial mycotic aneurysm in a case of infective endocarditis. Singapore Med J 1993;34:172-4.

12. Iacovelli F, Scicchitano P, Zanna D, Marangelli V, Favale S. Left ventricle outflow tract vegetation, embolism and troponin rise: an infective endocarditis case report. Intern Emerg Med 2012;7 Suppl 2:S145-7.

13. Hoen B, Duval X. Clinical practice. Infective endocarditis. N Engl J Med 2013;368:1425-33. 